\title{
Subcutaneous
}

\section{depo-medroxyprogesterone acetate}

\section{Sharon Cameron}

Consultant Gynaecologist, NHS Lothian, Sexual and Reproductive Health,

Chalmers Centre, Edinburgh, UK

\section{Correspondence to}

Dr Sharon Cameron, Sexual and Reproductive Health, NHS Lothian, Chalmers Centre, 21 Chalmers Street, Edinburgh EH3 9ES, UK; sharon.cameron@ed.ac.uk

Received 7 December 2012 Revised 31 January 2013 Accepted 31 January 2013 of Family Planning and Reproductive Health Care 2013;39:75-77.
To cite: Cameron S. Journal

\section{INTRODUCTION}

Since 2004, a micronised formulation of depo-medroxyprogesterone acetate (DMPA) that is delivered as a subcutaneous injection has been available. It is currently in clinical use in the USA and in nine countries throughout Europe. ${ }^{1}$ As of March 2013 this product is being marketed in the UK as Sayana ${ }^{\circledR}$ Press (Pfizer, UK). The indications and contraindications for the use of subcutaneous depo-medroxyprogesterone acetate (DMPA-SC) are the same as for the intramuscular preparation (DMPA-IM, Depo Provera $\left.{ }^{\circledR}\right) .{ }^{1}$ Although the total dose of DMPA is 30\% lower with the SC compared to the IM preparation (104 vs $150 \mathrm{mg}$ ), the efficacy and effect on the return of fertility are no different from the IM preparation ${ }^{2} 3$ (Table 1).

\section{PHARMACOKINETICS}

Compared to DMPA-IM, there is a slower rate of absorption of DMPA-SC with lower peak serum medroxyprogestrone acetate (MPA) concentrations providing a long duration of effect. Serum MPA levels still remain above the threshold for inhibition of ovulation for 3 months. $^{2}$ Based upon this, the recommended injection interval for DMPA-SC is $13 \pm 1$ weeks. ${ }^{1}$ The median time to return of ovulation after DMPA-SC is consistent with that for DMPA-IM; and at 12 months postinjection, studies show that ovulation will have resumed in $97 \%$ of subjects. ${ }^{2}$ Trough concentrations of DMPA-SC are not significantly different between injections given on the anterior abdominal wall or anterior thigh, suggesting that the injection site does not affect the contraceptive efficacy. ${ }^{2}$

\section{SAFETY AND EFFICACY}

Two large Phase III international trials (open, non-comparative) conducted in North and South America and Europe/ Asia assessed the 1-year contraceptive efficacy, safety and user satisfaction of DMPA-SC. ${ }^{3}$ There were no pregnancies observed in over 1700 women and over 16000 woman-cycles of exposure to DMPA-SC. Importantly, more than onequarter of subjects in both studies were either overweight or obese, demonstrating that the efficacy is not attenuated in women with high body mass index. ${ }^{3}$ This is supported by pharmacokinetic data
Table 1 Comparison of subcutaneous depo-medroxyprogesterone acetate (DMPA-SC) and intramuscular depo-medroxyprogesterone acetate (DMPA-IM)

\begin{tabular}{ll}
\hline Characteristic & DMPA preparation \\
\hline Recommended injection interval & DMPA-SC 13 weeks \\
& DMPA-IM 12 weeks (accepted interval up to 14 weeks) \\
Efficacy & DMPA-SC=DMPA-IM \\
Return to fertility & DMPA-SC=DMPA-IM \\
Bleeding pattern & DMPA-SC=DMPA-IM (60\% amenorrhoea at 12 months) \\
Metabolic effects & No evidence for difference \\
Weight gain & DMPA-SC=DMPA-IM \\
Bone mineral density loss & DMPA-SC=DMPA-IM (5\% loss at 2 years) \\
Side effects & Possibly less pain with new injection system (UnijectTM) for DMPA-SC \\
Administration & Approximately 1 in 10 users of DMPA-SC get injection site nodule \\
Single injection cost (UK) & Potential for self-administration of DMPA-SC \\
& May facilitate pharmacy administration \\
& DMPA-IM $£ 6.00$ (British National Formulary) \\
& DMPA-SC $£ 6.90$ (Department of Health, March 2013)
\end{tabular}


that show that in obese women medroxyprogesterone levels remain above the level needed to prevent ovulation. ${ }^{4}$ Satisfaction rates were high with DMPA-SC in these studies, with continuation rates at 1 year of $69 \%$ in the Americas and $81 \%$ in the Europe/Asia trial. ${ }^{3}$ The commonest reason in the trials for discontinuation was increased or irregular bleeding.

\section{EFFECT ON BONES, WEIGHT AND BLEEDING}

In a randomised controlled trial of 225 women using DMPA-SC or DMPA-IM for 2 years, changes in bone mineral density (BMD) at 2 years compared to baseline (hip and lumbar spine) were not significantly different and appeared to be reversible on stopping (approximately 5\% BMD loss). ${ }^{5}$ Furthermore, weight changes were similar in users of both DMPA-SC and DMPA-IM, with a median weight increase of $3.5 \mathrm{~kg}$ after 2 years. ${ }^{5}$ In the Phase III multinational trials the median weight gain was less than $2 \mathrm{~kg}$ at 1 year. $^{3}$

Bleeding patterns also appear to be comparable to those of users of DMPA-IM. ${ }^{5}$ In the first 3 months of use, high proportions of women (74\%) experience spotting and/or bleeding with DMPA-SC, but by 12 months of use over $60 \%$ of new users of DMPA-SC are amenorrhoeic. ${ }^{3}$ High rates of amenorrhoea have also been reported for established users of DMPA-IM who switch to DMPA-SC, ${ }^{6}$ with amenorrhoea rates in the region of $90 \%$ by the end of 1 year among those who continue to use it. ${ }^{6}$

\section{INJECTION SITE}

Injection site skin reactions are common; $8 \%$ of users report erythema or irritation at the site. ${ }^{5}$ In addition, skin changes such as dimpling or lumps at the injection sites have been reported to occur in approximately 1 in 10 users. $^{3}{ }^{6}$ These may represent a form of lipoatrophy, which is a recognised occurrence with other SC hormonal injections such as insulin and growth hormone. ${ }^{6}$ Resolution of lesions has been reported, ${ }^{6}$ but women need to be warned of this cosmetic side effect, as it is likely that this will limit acceptability of DMPA-SC for some.

\section{INJECTION SYSTEM AND TECHNIQUE}

DMPA-SC is available within a new simple delivery system known as Uniject ${ }^{\mathrm{TM}}$ (Figure 1). Essentially this comprises a polyethylene reservoir prefilled with DMPA and a fine SC needle. The single-dose container must be shaken vigorously before use so that the dose is given in a uniform suspension. The injection must be activated before use. This activation process involves piercing an internal seal so that the medicine can come out of the needle when the reservoir is squeezed. The injection is given over several seconds and with the needle pointing downwards. DMPA-SC is most commonly injected into the anterior thigh or anterior abdominal wall. ${ }^{1}$

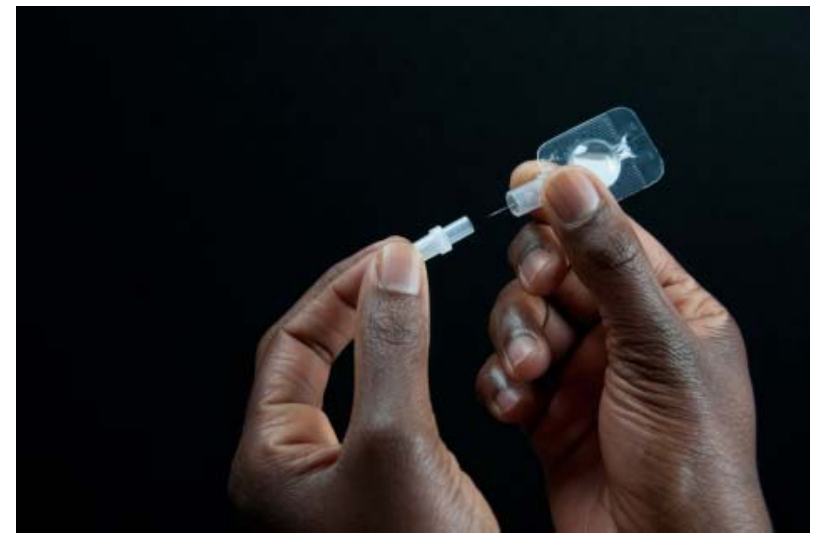

Figure 1 The Uniject ${ }^{\mathrm{TM}}$ injection system with a short needle for subcutaneous contraceptive injection. Figure (C) PATH/Patrick McKern reproduced with kind permission.

The availability of DMPA-SC may facilitate administration of injectable contraception from a range of health professionals, including community pharmacists. This has already been shown to be feasible in a pilot study in the USA. ${ }^{7}$

Since SC injections employ a finer needle than is used for IM injections, one might expect that women would find DMPA-SC less painful than DMPA-IM. However, published studies have shown that most users rate pain associated with DMPA given by either the SC or IM route as 'low' and no significant differences in reported levels of pain could be elicited..$^{5} 6$ However, existing studies did not use the new Uniject delivery system for DMPA-SC and so it is possible that that woman may find the SC injection given with the Uniject system even more acceptable.

\section{SELF-ADMINISTRATION}

Since it is administered subcutaneously, DMPA-SC offers the possibility of self-administration, which may be attractive for women. The need to attend a clinic every 3 months to receive an injection is also a disadvantage (and cost) for the UK National Health Service. Currently in the UK most women do not need to visit their contraceptive provider more than once a year to obtain supplies of contraceptive pills, and for users of implants and intrauterine contraception a visit may be required only for replacement after 3 and 5 years, respectively. In a survey of Scottish women, two-thirds of current users of DMPA-IM expressed a theoretical preference to use a preparation that they could self-inject at home. ${ }^{8}$ In the same survey, a significant proportion of ex-users or never-users of the injectable said that they would consider using this method if they were able to self-inject. ${ }^{8}$

To date, there have been three studies of selfadministration of DMPA-SC. ${ }^{6}{ }^{10}$ In two studies, conducted in the USA (50 women) and the UK (64 women), women were taught to self-inject, and when 
deemed competent gave themselves their first injection under the supervision of the study co-ordinator, and were then given a further three supplies (equivalent to 1 year of contraception) to take home to selfadminister. ${ }^{6}{ }^{9}$ In both studies continuation rates with the method were high at the end of 1 year $(74 \%$ and $88 \%$ in the USA and UK, respectively). In both studies most women $(87 \%$ and $90 \%)$ reported that selfadministration was easy and that they would recommend the method to a friend (94\% and 95\%). ${ }^{6}$ The third study showed that self-administration of DMPA-SC was feasible even in teenagers after brief training. ${ }^{10}$ In all studies conducted to date the DMPA-SC that was used was packaged as a prefilled syringe with a separate SC needle that needed to be attached to the syringe. Training women to selfadminister using this DMPA-SC delivery system (which is arguably more complicated than the Uniject system) was estimated to take less than 30 minutes. ${ }^{6} 9$

The possibility to self-administer DMPA-SC may not only be attractive to women in developed countries, but also to women in developing countries, where there is a huge unmet need for contraception. The ability to self-administer DMPA-SC offers the potential to enhance safe, effective contraception for women in these countries.

\section{WHAT DOES DMPA-SC ADD?}

The availability of DMPA-SC in the UK is welcome, as it increases the choice of contraception for women. SC delivery may render the injection more acceptable to some women, and should also facilitate provision from a wider range of health professionals such as community pharmacists. If DMPA-SC can be approved for self-administration (it is not currently licensed for this), which has been shown to be a desirable and feasible option, then this would make the method more convenient for women. By increasing contraceptive choice and improving convenience of the injectable, DMPA-SC has a potentially important role to play in improving uptake and continuation rates of effective methods of contraception. Ultimately this could translate into fewer unintended pregnancies with their associated emotional distress for women and financial cost to the health care system.

Competing interests The author received an investigator-initiated research grant from Pfizer to conduct the study of self-administration of DMPA-SC and has recently been member of an advisory group on this product.

Provenance and peer review Commissioned; externally peer reviewed.

\section{REFERENCES}

1 Public assessment report. Mutual recognition procedure. Sayana $104 \mathrm{mg} / 0.65 \mathrm{ml}$ suspension for injection. UK/H/0960/ 001/MR. http://www.mhra.gov.uk/home/groups/par/ documents/.../con088109.pdf [accessed 5 December 2012].

2 Jain J, Dutton C, Nicosia A, et al. Pharmacokinetics, ovulation suppression and return to ovulation following a lower dose subcutaneous formulation of Depo-Provera ${ }^{\circledR}$. Contraception 2004;70:11-18.

3 Jain J, Jakimiuk AJ, Bode FR, et al. Contraceptive efficacy and safety of DMPA-SC. Contraception 2004;70:269-275.

4 Segall-Gutierrez P, Du J, Niu C, et al. Effect of subcutaneous depo-medroxyprogesterone acetate (DMPA-SC) on serum androgen markers in normal-weight, obese, and extremely obese women. Contraception 2012;86:739-745.

5 Kaunitz AM, Darney PD, Ross D, et al. Subcutaneous DMPA vs. intramuscular DMPA: a 2-year randomized study of contraceptive efficacy and bone mineral density. Contraception 2009;80:7-17.

6 Cameron ST, Glasier A, Johnstone A. Pilot study of home self-administration of subcutaneous depo-medroxyprogesterone acetate for contraception. Contraception 2012;85:458-464.

7 Picardo C, Ferreri S. Pharmacist-administered subcutaneous depot medroxyprogesterone acetate: a pilot randomized controlled trial. Contraception 2010;82:160-167.

8 Lakha F, Henderson C, Glasier A, et al. The acceptability of self-administration of subcutaneous Depo-Provera. Contraception 2005;72:14-18.

9 Prabhakaran S, Sweet A. Self-administration of subcutaneous depot medroxyprogesterone acetate for contraception: feasibility and acceptability. Contraception 2012;85:453-457.

10 Williams RL, Hensel DJ, Fortenberry JD. Self-administration of subcutaneous depot medroxyprogesterone acetate by adolescent women. Contraception 2013; 4 January 2013. doi: pii: S0010-7824(12)01027-X. 10.1016/j.contraception.2012. 11.019 [Epub ahead of print].

\section{JOURNAL REVIEW WRITERS}

The Journal regularly reviews articles from related publications and is looking to recruit more volunteers to compile these short reviews. For further information and/or to offer your services (at most once or twice a year) please e-mail your contact details to the Journal Editorial Office together with a note of any special interests and/or expertise to assist us in allocating relevant articles to individual reviewers. For further information on this role please contact the Journal Editorial Office at journal@fsrh.org. 